\title{
Unilateral Choanal Atresia in Adult: Case Report
}

\author{
Dr. Vishwambhar Singh
}

Senior Resident, department of Otorhinolaryngology, Institute of Medical Sciences, Banaras Hindu University, Varanasi.

\begin{abstract}
Unilateral choanal atresia usually presents later in life than bilateral and may present in adults. Here is a case report of a 19 year old male who presented with complains of unilateral nasal obstruction and discharge not clearly pointing to the diagnosis. The canalization was done endoscopically. This article focus the role of CT scan and nasal endoscopy in the diagnosis and treatment of the same.
\end{abstract}

Keywords: Choanal atresia, CHARGE, nasobuccal membrane, nasal obstruction, Endoscopy

\section{Introduction}

Choanal atresia is defined as a developmental failure of the posterior nasal cavity to communicate with the nasopharynx. Choanal atresia has an incidence of 1 in 5000 to 7000 births ${ }^{1}$, occurs more commonly in females than in males and is more often unilateral than bilateral ${ }^{2}$. Bilateral choanal atresia requires rapid management at birth and prompt diagnosis because neonates are predominantly obligate nasal breathers ${ }^{3}$. Unilateral choanal atresia is often diagnosed after several months of life. Unilateral choanal atresia is usually not associated with other facial anomaly or with syndromic malformations although most common associated congenital anomaly is CHARGE association $(\mathrm{C}=$ coloboma, $\mathrm{H}=$ heart disease, $\mathrm{A}=$ atresia of choanae, $\mathrm{R}=$ retarded growth and development, $\mathrm{G}=$ genital hypoplasia, $\mathrm{E}=$ ear deformities or deafness) ${ }^{4}$. Persistent unilateral rhinorrhea or failure to pass a catheter through the obstructed nasal fossa (during a general anaesthesia for example) makes the diagnosis of choanal atresia probable. Choanal atresia is an uncommon and rarely recognized cause of unilateral nasal obstruction. This case report documents the case of a patient with unilateral choanal atresia who remained undiagnosed for many years.

\section{Case Report}

A 19 year old man presented with a prolonged history of complete nasal obstruction and discharge on left side. He also complained of loss of sense of smell on left side since childhood with associated features of mouth breathing and snoring. On examination he had slight facial asymmetry with the dorsum slightly deviated to right.

On speculum examination initially it was confused as a case of chronic rhinosinusitus as thick vicid secretion could be seen at the floor of nose. On x-ray PNS only hazy bilateral maxillary sinuses could be noted.

Patient was taken for endoscopic examination, which on suction of the left nasal cavity on first pass nasal endoscopy showed absence of posterior choana, which was confirmed on Rhinography and CT-scan. It was finally diagnosed a case unilateral bony choanal atresia. This patient was treated multiple times before at many general practitioners as a case of allergic chronic rhinosinusitis.
Computed tomography coronal cuts elicited bony atresia with prolapsed mucosal thickening at the left choana with polypoidal mass at posterior choanae region. Rest of the investigations where within normal limits.

Patient was posted for transnasal surgery. Endoscopic transnasal route was preferred, with initial use of burr and later enlarged using dilators. A pack was placed pre operatively in nasopharynx to protect the structures in the area. Silastic tube was inserted for six weeks to maintain the patency. Antibiotics, anti-inflammatory, saline nasal spray and proper nasal toileting was advised and the patient made an uneventful post-operative recovery.

\section{Discussion}

Choanal atresia is a congenital obstruction of posterior nasal aperture or choana, usually thought to be secondary to persistence of either the nasobuccal membrane of Hochstetter or the bucco-pharyngeal membrane from the foregut. This membrane normally ruptures between the fifth and sixth weeks of gestation to produce choanae. Failure of this membrane to rupture causes atresia of choanae and may be bony, membranous or mixed ${ }^{5}$. Previously reports suggested a $90 \%$ bony stenosis and $10 \%$ membranous, but more recent analysis suggests a mixed bony/membranous in $70 \%$ and pure bony in $30 \%{ }^{6}$.

Unilateral or bilateral choanal atresia was first described by Roederer in 1751 and was first reported in Britain in 1881 by Ronaldson .Carl Emmert in Bern operated successfully on a patient of choanal atresia in 1851 .He perforated the bilateral choanal atresia via the transnasal approach using a curved trocar after having practiced the perforating force on the hard palate of child's corpse. Bilateral choanal atresia presents at birth as a respiratory emergency. Occasionally unilateral choanal atresia may present in young with feeding difficulties especially when the non-affected side of the face is occluded. Unilateral cases do not present until late childhood or adulthood.

Anomalies associated with choanal atresia include polydactyly, nasal-auricular and palatal deformities, Crouzon's syndrome, Down syndrome, TreacherCollinssyndrome, DiGeorge syndrome, craniosynostosis, microencephaly, meningocele, meningoencephalocele, facial asymmetry, hypoplasia of the orbit and midface, cleft palate, and hypertelorism ${ }^{7}$. Retrospective review of medical 


\section{International Journal of Science and Research (IJSR) \\ ISSN (Online): 2319-7064 \\ Index Copernicus Value (2013): 6.14 | Impact Factor (2014): 5.611}

comorbid conditions of 78 children with choanal atresia found that common medical problems were otitis media with effusion (32\%), upper and lower airway diseases $(32 \%$ and $23 \%$, respectively), cardiac anomalies (19\%), and gastrointestinal tract disorders $(18 \%)^{8}$. Statistically significant correlations were found for bilateral choanalatresia and cardiac disorders, CHARGE syndrome, obstructive sleep apnea, hematological problems, and prematurity or failure to thrive. Generally, $65 \%$ to $75 \%$ of patients with choanalatresia are unilateral, whereas the rest are bilateral ${ }^{9}$. About $30 \%$ are pure bony, whereas $70 \%$ are mixed bony-membranous ${ }^{10}$. Theatretic plate is usually sited in front of the posterior end of the nasal septum. The anatomic deformities include a narrow nasal cavity, lateral bony obstruction by the lateral pterygoidplate, medial obstruction caused by thickening of the vomer, and membranous obstruction ${ }^{11}$. Acquired posterior choanal atresia rarely occurs. It is usually caused by rhinopharyngeal injury e.g. after adenoidectomy; radiotherapy for nasopharyngeal carcinoma ${ }^{12}$; tuberculosis or syphilis of epipharyn ${ }^{13}$, or sometimes by unknown causes.

\section{Conclusion}

Choanal atresia can be an isolated anomaly, however $60 \%$ cases of congenital defect has found to be associated with Down's and Treacher Collin's syndrome but may be found with other isolated defects such as micrognathia, tracheoesophageal fistula, cleft and high arched palate, missing teeth and facial cleft. In addition to these random associations choanal atresia has recently been linked with a number of specific defects the so called CHARGE association. Our reported case highlighted the fact that choanal atresia especially unilateral is a rarely recognised cause of nasal obstruction and is often diagnosed late. Hence endoscopic/radiological investigations are mandatory to make a diagnosis for complaints of persistent nasal obstruction for so many years.

\section{References}

[1] Samadi DS, Shah UK, Handler SD. Choanal atresia: a twenty years review of medical co-morbidityand surgical outcomes. Laryngoscope 2003:113:254-8

[2] Dobrowski JM, Grundfast KM, Rosenbaum KN, Zajtchuk JT. Otorhinolaryngic manifestations ofCHARGE association. Otolaryngol Head Neck Surg 1985; 93: 798-803.

[3] Karampal Singh, SarlaHooda, PunamRaghove, GeetaAhlawat, RashmiTaneja: Bilateral Congenital Choanal Atresia: A Case Report. The Indian Anaesthetists' Forum, July 2011(2):http://www.theiaforum.org

[4] Bergstrom L, Owens O. Posterior choanal atresia: a syndromal disorder. Laryngoscope 1984;94:1273-6.

[5] Dunham ME, Miller RP. Bilateral choanal atresia associated with malformation of the anterior skull base: embryogenesis and clinical implications. Ann OtolRhinolLaryngol 1992; 101: 916-9.

[6] ParayaAssanasen, ChoakchaiMetheetrairut. Choanal Atresia.J Med Assoc Thai 2009; 92 (5): 699-706: http://www.mat.or.th/journal.
[7] Harris J, Robert E, Kallen B. Epidemiology of choanal atresia with special reference to the CHARGE association. Pediatrics 1997; 99: 363-7.

[8] Samadi DS, Shah UK, Handler SD. Choanal atresia: a twenty-year review of medical comorbidities and surgical outcomes. Laryngoscope 2003; 113: 254-8.

[9] Dobrowski JM, Grundfast KM, Rosenbaum KN, Zajtchuk JT. Otorhinolaryngic manifestations of CHARGE association. Otolaryngol Head NeckSurg 1985; 93: 798-803.

[10] Brown OE, Pownell P, Manning SC. Choanal atresia: a new anatomic classification and clinical management applications. Laryngoscope 1996; 106: 97-101.

[11] Harner SG, McDonald TJ, Reese DF. The anatomy of congenital choanal atresia. Otolaryngol Head Neck Surg 1981; 89: 7-9.

[12] Ku PK, Tong MC, Tsang SS, van Hasselt A. Acquired posterior choanal stenosis and atresia: management of this unusual complication after radiotherapy for nasopharyngeal carcinoma. Am J Otolaryngol 2001; 22: 225-9.

[13] Imoto T, Hashimoto K. A case report of nasopharyngeal tuberculosis resulted in nasopharyngeal obstruction. Otol Fukuoka 1981; 27: 592-4.

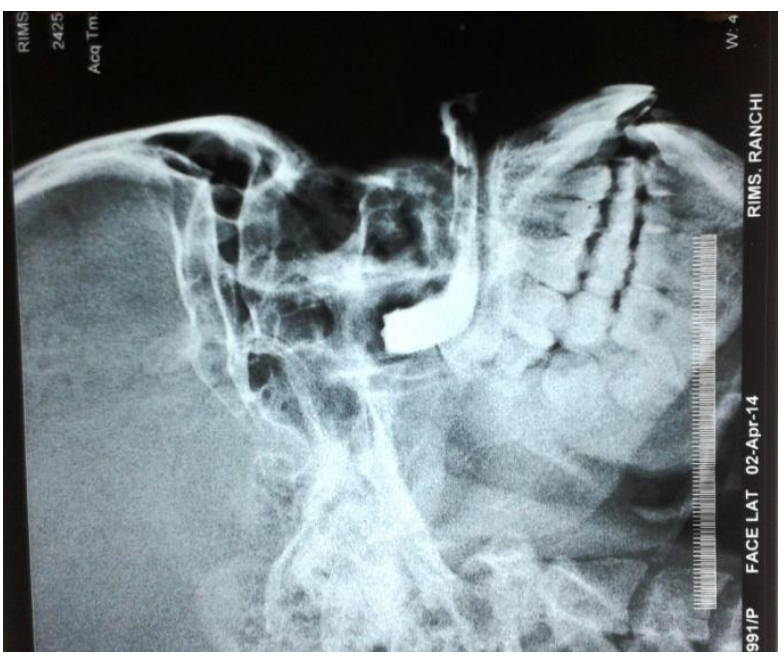

Figure 1: Rhinography

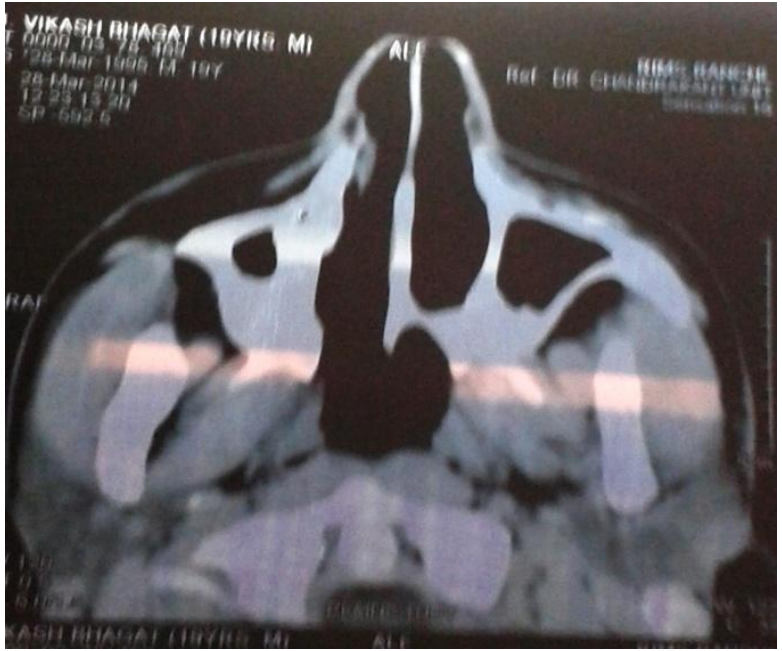

Figure 2: CT-Scan showing bony atresia 


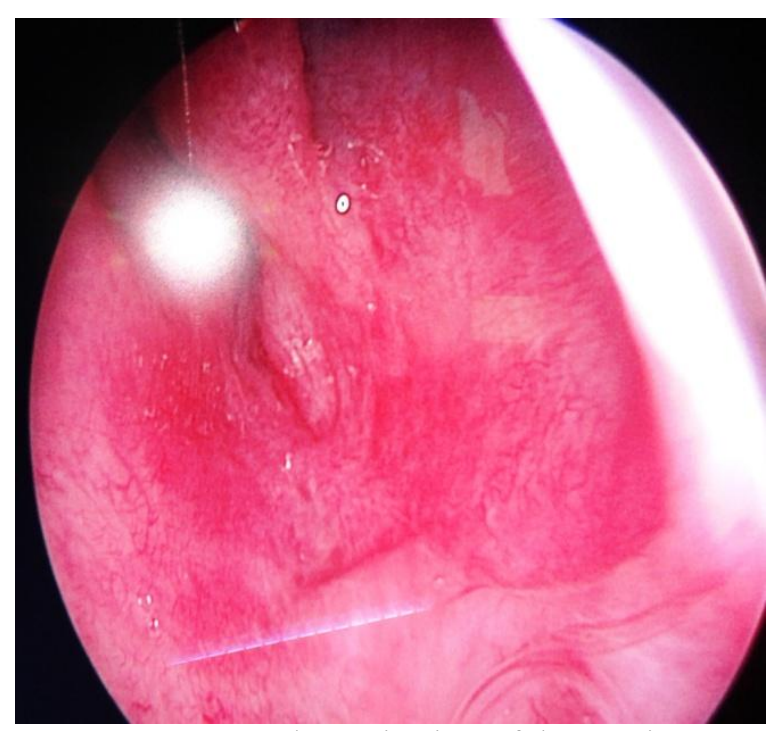

Figure 3: endoscopic view of the atresia

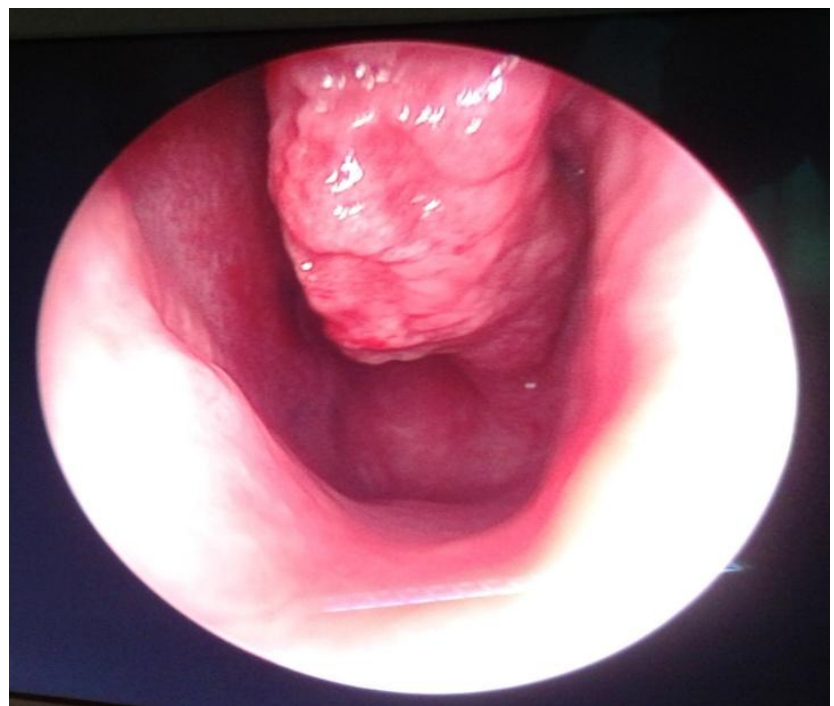

Figure 4: Endoscopic view of the atresia with inferior turbinate 